\title{
A comparison of different methods for including 'age at menopause' in analyses of the association between hormone replacement therapy use and breast cancer
}

\author{
Julie A Simpson, Dallas R English, Robert J MacInnis, Dorota M Gertig, John L Hopper, Graham G Giles
}

\begin{abstract}
Background and methodology Late 'age at menopause' is a recognised risk factor for postmenopausal breast cancer and is also associated with decreased use of hormone replacement therapy (HRT). When investigating the association between HRT use and breast cancer risk it is therefore necessary to adjust for the potential confounder, 'age at menopause'. 'Age at menopause', however, cannot be determined for women with a hysterectomy and ovarian conservation. Using data on 13357 postmenopausal women in whom 396 cases of invasive breast cancer were diagnosed during 9 years of follow-up from the Melbourne Collaborative Cohort Study, we compared the estimates of relative risk of HRT use for breast cancer for three different methods of dealing with missing data: complete-case analysis, single imputation and multiple imputation.
\end{abstract}

Results 'Age at menopause' was missing for $17 \%$ of the data. Both HRT use and 'age at menopause' were

\section{Introduction}

When investigating the association between use of hormone replacement therapy (HRT) and breast cancer risk, the analysis should include adjustment for the potential confounder 'age at menopause'. Historically, 'age at menopause' is the age when a woman no longer menstruates, and this is often termed the age at natural menopause. Women who have undergone a hysterectomy (a common surgical procedure) prior to when their periods would have stopped naturally have their uterus removed together with both, one or none of the ovaries. For women with the uterus and both ovaries removed (i.e. a bilateral oophorectomy), the 'age at menopause' is the age at surgery. For women with the uterus and one or none of the ovaries removed, which we will term a 'simple hysterectomy', the 'age at menopause' cannot be defined. This is because the women are no longer menstruating but still have functioning ovaries after surgery.

There are different approaches adopted for the above problem of missing data in analyses of observational studies investigating the association between HRT and breast

Cancer Epidemiology Centre, The Cancer Council Victoria, Melbourne and the Centre for Molecular, Environmental, Genetic and Analytic Epidemiology, University of Melbourne, Melbourne, Australia

Julie A Simpson, PhD, Statistican

Dallas R English, PhD, Epidemiologist

Robert J Maclnnis, PhD, Statistican

Graham G Giles, PhD, Epidemiologist

Centre for Molecular, Environmental, Genetic and Analytic Epidemiology, University of Melbourne, Melbourne, Australia Dorota M Gertig, PhD, Epidemiologist John L Hopper, PhD, Epidemiologist

Correspondence to: Dr Julie A Simpson, Centre for Molecular, Environmental, Genetic and Analytic Epidemiology, School of Population Health, University of Melbourne, Level 2/723 Swanston Street, Carlton, VIC 3053, Australia.

E-mail: julieas@unimelb.edu.au significant risk factors for breast cancer, although 'age at menopause' only marginally confounded the estimates of risk for HRT. Women with 'age at menopause' missing did not represent a random sample of the population. Complete-case analyses resulted in higher estimates of the risk associated with HRT use compared with the different methods of imputation.

Discussion and conclusions We recommend that analyses investigating the association between HRT and breast cancer should present the results in two ways: excluding women with 'age at menopause' missing and including the women using multiple imputation. For both methods, estimates of risk, with and without the adjustment of 'age at menopause', should be given.

Keywords age at menopause, hormone replacement therapy, imputation, missing data

J Fam Plann Reprod Health Care 2007; 33(1): 11-16 (Accepted 10 July 2006)
Key message points

- When investigating the association between hormone replacement therapy (HRT) use and breast cancer risk it is necessary to adjust for the potential confounder, 'age at menopause'. However, 'age at menopause' cannot be determined for women with a hysterectomy and ovarian conservation.

- Large observational studies investigating the association between HRT and breast cancer have adopted different approaches. Some studies have excluded women with a hysterectomy and ovarian conservation from the analysis, whereas others have included the women by assigning a fixed 'age at menopause'.

- We recommend that the risk of breast cancer associated with HRT be presented in two ways: excluding women with a hysterectomy and ovarian conservation, and including the women using multiple imputation. For both methods, the estimates of risk, with and without the adjustment of 'age at menopause', should be presented to show the extent of confounding.

cancer. In a pooled analysis of more than 50 epidemiological studies ${ }^{1}$ all postmenopausal women with an unknown 'age at menopause' were excluded, whereas in the Million Women Study (MWS) ${ }^{2}$ women with an unknown 'age at menopause' were included by assigning a time since menopause similar to that of women of a similar age.

Pike et al. ${ }^{3}$ have shown, using a hypothetical example, that the common practice of assigning a particular 'age at menopause' (e.g. 55 years) for all women with simple hysterectomy (known as single imputation) results in attenuated estimates of the relative risk of HRT use compared with the estimates obtained when the women with simple hysterectomy are excluded. This hypothetical example was confirmed with data from the Nurses' Health Study ${ }^{4}$ and from the pooled analysis of more than 50 epidemiological studies. ${ }^{1}$ In the MWS,${ }^{2}$ however, the point estimates for the relative risk of breast cancer in current and past users of HRT varied by less than $1 \%$ when a 
sensitivity analysis was performed that excluded women with 'age at menopause' missing.

In the papers by Pike et al. ${ }^{3}$ and Rockhill et al..$^{4}$ it was assumed that the so-called 'complete-case analysis' (i.e. exclusion of women missing 'age at menopause') gives unbiased estimates of the true risk of HRT for breast cancer, and hence the lower risk from assigning a fixed age at menopause was considered to be an underestimate. However, excluding these women may also have produced biased estimates if women with simple hysterectomy did not represent a random subsample of the data. $5,6 \mathrm{In}$ addition, the statistical power of such analyses will be reduced by excluding subjects.

An alternative to excluding the women with 'age at menopause' missing would be to use the methodology called multiple imputation. Multiple imputation has advantages over single imputation methods because: it allows variation in the 'age at menopause', any type and any number of variables can be used in the imputation procedure, and it gives the most robust variance estimates. $^{7}$

In this paper, we have applied multiple imputation to a prospective study of breast cancer and compared the estimates of relative risk of HRT use for breast cancer between the multiple imputation method, other single imputation techniques and complete-case analysis.

\section{Methods}

\section{Study subjects}

The data used in this paper came from 13447 postmenopausal women who were participants of the Melbourne Collaborative Cohort Study (MCCS). The MCCS is a prospective cohort study comprising 41528 people (24 479 women) aged between 25 and 75 years at baseline, $99.3 \%$ of whom were aged $40-69$ years. Men and women volunteers were recruited from the Melbourne metropolitan area in the early 1990s. Details of the MCCS have been published elsewhere. ${ }^{8}$

\section{Outcome of interest}

Diagnosis of invasive breast cancer was the outcome of interest. Breast cancers were identified from the population-complete Victorian Cancer Registry. Follow-up began at the age at baseline (attendance for interview) and ended (censoring) at the age of breast cancer diagnosis, age of death, the age left Victoria, or the age at 30 June 2002, whichever came first. The average follow-up was approximately 9 years. Women with in situ breast cancers were not included as cases.

\section{Predictor variables}

The potential predictors of breast cancer risk that we investigated were HRT use (never, past, current users), age at menopause (years), age at baseline (years), education level (none/primary, secondary/trade, tertiary), age at menarche $(<13,13,13+$ years $)$, age at first live birth and parity combined (nulliparous, age at first live birth $<25$ years and parity equals one, age at first live birth $<25$ years and parity $>1$, age at first live birth $\geq 25$ years and parity equals one, age at first live birth $\geq 25$ years and parity $>1$ ), smoking status (never, past, current), alcohol intake $(0$, $1-19,20-39,40+\mathrm{g} /$ day), body mass index (BMI; $\mathrm{kg} / \mathrm{m}^{2}$ ) and country of birth (Australia, UK, Greece/Italy). All of the above variables were recorded at baseline. No other additional variables, that were not potential predictors of breast cancer, were considered a priori to be associated with missing 'age at menopause'.

'Age at menopause' was determined as the age at which a woman's periods had ceased naturally for at least the past
12 months (age at natural menopause) or, if she had a hysterectomy before her periods stopped naturally, the age at which she had a bilateral oophorectomy (age at surgical menopause). The remaining women, with simple hysterectomy, were considered postmenopausal with unknown 'age at menopause' if their plasma concentration of estradiol was measured ( $10 \%$ of the women) and was less than $109 \mathrm{pmol} / \mathrm{l}$ (the cut-off level at which $90 \%$ of women were correctly classified as premenopausal or naturally postmenopausal in the cohort) or their age at baseline was greater than 55 years (the age at which natural menopause had occurred in $90 \%$ of the total cohort).

\section{Investigation of the missing data}

The relationship between missing 'age at menopause' and breast cancer was examined using Kaplan Meier curves stratified by a missing value indicator. The log rank test was used to test for a difference in the probability of breast cancer at any time of follow-up for the missing and nonmissing data. The relationship between 'missingness' (yes vs no) and the predictor variables was assessed using logistic regression.

\section{Multiple imputation}

In brief, five sets of data were imputed for the women with 'age at menopause' missing from a model that included all of the predictor variables mentioned above and the outcome variable, diagnosis of breast cancer (yes/no). For a more detailed explanation of multiple imputation refer to the Appendix.

\section{Statistical software}

Multiple imputation was carried out using SPLUS 6.2 (Insightful Corporation, Seattle, WA, USA). Combining the results of the analyses of the five complete datasets (imputed plus observed data) using Rubin's rules was performed in Stata/SE 8.0 (Stata Corporation, College Station, TX, USA) using the programmes written by Carlin et al. ${ }^{9}$ All other statistical analyses were performed using Stata/SE 8.0.

\section{Results}

In the MCCS there were 13447 postmenopausal women in whom 398 invasive breast cancer cases were diagnosed during follow-up. The incidence rate of breast cancer was 3.28 per 1000 woman-years. Approximately $6 \%$ of the women had either died or moved within the time of follow-up.

\section{Missing data}

The proportion of missing data ranged from $0 \%$ to $0.3 \%$ for all variables except 'age at menopause' where $17.5 \%$ of data were missing. For the purpose of this paper, therefore, data were imputed only for 'age at menopause' since the amount of missing data in the other variables was negligible. The final dataset comprised 13357 women (396 breast cancer cases) with complete data for all variables in the model except 'age at menopause', which was unknown for $2322(17.4 \%)$ women. Ninety-nine percent of the 2322 women with 'age at menopause' missing had a simple hysterectomy before menopause. The mean \pm standard deviation (SD) observed 'age at menopause' was: $49.6 \pm$ 4.5 years for never users $(n=8227), 49.1 \pm 5.4$ for past users $(n=1143)$, and $48.1 \pm 5.9$ for current users $(n=1665)$ of HRT.

The probability of breast cancer at any time point during follow-up was similar for women with 'age at menopause' missing and not missing (log rank test, $p=$ 0.64). Additionally, the type of menopause (natural, 
Table 1 Distribution of predictor variables by 'age at menopause' missing

\begin{tabular}{|c|c|c|c|}
\hline \multirow[t]{2}{*}{ Predictor variables } & \multicolumn{2}{|l|}{ Age at menopause } & \multirow{2}{*}{$\begin{array}{l}\text { Multivariate odds } \\
\text { ratio }(95 \% \mathrm{Cl})^{\mathrm{a}}\end{array}$} \\
\hline & $\begin{array}{l}\text { Missing } \\
(n=2322)\end{array}$ & $\begin{array}{l}\text { Not missing } \\
(n=11035)\end{array}$ & \\
\hline \multicolumn{4}{|l|}{ HRT (\%) } \\
\hline Never user & 61.9 & 74.6 & 1.00 \\
\hline Past user & 14.9 & 10.4 & $0.55(0.48-0.63)$ \\
\hline Current user & 23.2 & 15.1 & $0.44(0.39-0.50)$ \\
\hline \multicolumn{4}{|l|}{ Education (\%) } \\
\hline None/primary & 20.0 & 27.1 & 1.00 \\
\hline Secondary/trade & 67.5 & 58.1 & $0.90(0.76-1.06)$ \\
\hline Tertiary & 12.5 & 14.8 & $1.10(0.89-1.35)$ \\
\hline \multicolumn{4}{|l|}{ Age at menarche (\%) } \\
\hline$<13$ years & 36.7 & 33.9 & 1.00 \\
\hline 13 years & 24.3 & 25.0 & $1.13(1.00-1.27)$ \\
\hline $13+$ years & 39.0 & 41.1 & $1.13(1.01-1.25)$ \\
\hline \multicolumn{4}{|l|}{ Age at first birth and parity combined (\%) } \\
\hline Nulliparous & 9.8 & 12.5 & 1.00 \\
\hline Single birth, $<25$ years & 15.6 & 13.9 & $0.62(0.51-0.75)$ \\
\hline Multiple birth, $<25$ years & 37.4 & 28.1 & $0.55(0.47-0.65)$ \\
\hline Single birth, $25+$ years & 19.1 & 24.6 & $0.93(0.78-1.11)$ \\
\hline Multiple birth, $25+$ years & 18.2 & 21.0 & $0.92(0.77-1.11)$ \\
\hline \multicolumn{4}{|l|}{ Smoking status (\%) } \\
\hline Never & 71.0 & 72.5 & 1.00 \\
\hline Past & 21.8 & 19.6 & $1.06(0.88-1.27)$ \\
\hline Current & 7.2 & 7.9 & $1.00(0.89-1.13)$ \\
\hline \multicolumn{4}{|l|}{ Alcohol intake (g/day) (\%) } \\
\hline None (0) & 48.8 & 49.1 & 1.00 \\
\hline Low (1-19) & 41.1 & 41.2 & $1.07(0.96-1.18)$ \\
\hline Medium (20-39) & 7.8 & 7.5 & $1.04(0.86-1.25)$ \\
\hline High $(40+)$ & 2.3 & 2.2 & $1.03(0.75-1.42)$ \\
\hline $\mathrm{BMI}\left(\mathrm{kg} / \mathrm{m}^{2}\right)($ mean $\pm \mathrm{SD})$ & $27.3 \pm 4.9$ & $27.3 \pm 4.9$ & 0.99 (0.98-1.00) (continuous) \\
\hline \multicolumn{4}{|l|}{ Country of birth (\%) } \\
\hline Australia & 75.5 & 66.7 & 1.00 \\
\hline UK & 7.2 & 6.1 & $0.92(0.77-1.10)$ \\
\hline Greece/ltaly & 17.3 & 27.2 & $1.31(1.10-1.56)$ \\
\hline $\begin{array}{l}\text { Age at baseline (years) (mean } \pm \text { SD) } \\
\text { Incidence rate of breast cancer }(95 \% \mathrm{CI})\end{array}$ & $\begin{array}{l}61.9 \pm 4.2 \\
3.29 \text { per } 1000 \text { person- } \\
\text { years }(2.60-4.18)\end{array}$ & $\begin{array}{l}60.1 \pm 5.8 \\
3.29 \text { per } 1000 \text { person- } \\
\text { years }(2.95-3.66)\end{array}$ & 0.93 (0.92-0.94) (continuous) \\
\hline
\end{tabular}

aDependent variable equals 'age at menopause' not missing (coded as ' 1 ') vs missing (coded as '0').

BMI, body mass index; SD, standard deviation.

bilateral oophorectomy and simple hysterectomy before menopause) did not modify the risk of breast cancer associated with HRT (likelihood ratio test, $p=0.21$ ). The distribution of the predictor variables by the dichotomous variable, 'age at menopause' missing (yes/no), are presented in Table 1. This shows that current and past users of HRT compared with never users $(p<0.001)$, an age at menarche of less than 13 years compared with $13+$ years $(p$ $=0.03$ ), less than 25 years of age for birth of first child compared with nulliparous $(p<0.001)$, Australian born compared with Greek/Italian born $(p=0.002)$ and an older age at baseline were associated with missing 'age at menopause' $(p<0.001)$. These differences indicate that a complete-case analysis may generate biased results.

\section{Imputed data}

Data from the $840(6.3 \%)$ women who had undergone a bilateral oophorectomy were excluded from the imputation model. Although the means of the imputed values for 'age at menopause' were consistent with the observed data for each of the five imputed datasets (Table 2), the proportions of subjects within specific age categories and the minimum ages were different. Further investigation showed that the observed values for 'age at natural menopause' were not Normally distributed but slightly negatively skewed with evidence of digit preference (Table 2, Figure 1).

\section{Estimates of breast cancer risk associated with HRT use and 'age at menopause'}

Table 3 presents the estimated risk for breast cancer associated with HRT use and 'age at menopause'. The results of six different Cox regression models are presented. The first two models exclude women with 'age at menopause' missing and so are complete-case analyses $(n=11035)$. The third model includes data from all of the women $(n=13357)$ but does not adjust for 'age at menopause'. Models 4 to 6 include data from all of the women $(n=13357)$ and adjust for 'age at menopause'. Model 4 uses multiple imputation, Model 5 uses single imputation (each woman assigned 55 years) for those women with 'age at menopause' missing, and Model 6 uses single imputation by assigning the age at

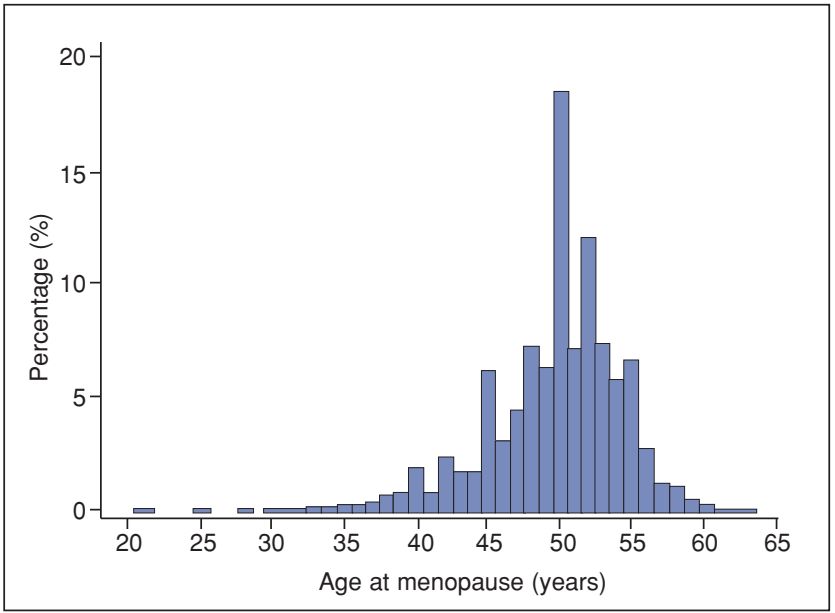

Figure 1 Histogram of observed 'age at menopause' for women with a natural menopause $(n=10195)$ 
Simpson et al.

Table 2 Comparison of the distribution of the imputed values and observed values for 'age at menopause' (840 women with a bilateral oophorectomy were excluded from the imputation model)

\begin{tabular}{|c|c|c|c|c|c|}
\hline \multirow[t]{2}{*}{ Dataset } & \multicolumn{5}{|c|}{ Age at menopause (years) } \\
\hline & Mean \pm SD (range) & $<45$ & 45-49 & $50-54$ & $55+$ \\
\hline $\begin{array}{l}\text { Observed values for women with a natural } \\
\text { menopause }(n=10195)\end{array}$ & $49.8 \pm 4.3(21-63)$ & $11 \%$ & $27 \%$ & $51 \%$ & $12 \%$ \\
\hline \multicolumn{6}{|l|}{ Imputed values $(n=2322)$} \\
\hline Dataset 1 & $50.5 \pm 4.7(24-66)$ & $10 \%$ & $28 \%$ & $43 \%$ & $19 \%$ \\
\hline Dataset 2 & $50.4 \pm 4.6(24-65)$ & $10 \%$ & $30 \%$ & $42 \%$ & $18 \%$ \\
\hline Dataset 3 & $50.4 \pm 4.6(24-64)$ & $10 \%$ & $29 \%$ & $45 \%$ & $16 \%$ \\
\hline Dataset 4 & $50.4 \pm 4.6(24-64)$ & $9 \%$ & $31 \%$ & $43 \%$ & $17 \%$ \\
\hline Dataset 5 & $50.5 \pm 4.6(24-64)$ & $10 \%$ & $30 \%$ & $43 \%$ & $18 \%$ \\
\hline
\end{tabular}

hysterectomy as the 'age at menopause' for those women where the 'age at menopause' is missing. For 255 of the women with simple hysterectomy the age at hysterectomy was unknown, and therefore these women were excluded from the analysis.

In all models, current use of HRT and a later 'age at menopause' were statistically significant risk factors for invasive breast cancer. For the complete-case analysis (11 035 women), the estimates of risk associated with both current and past HRT use were only slightly increased when 'age at menopause' was included as a confounder but were much higher compared with the estimates generated from data of all 13357 women, irrespective of the inclusion or exclusion of 'age at menopause' in the model. Assuming the estimate obtained from multiple imputation was unbiased, the percentage relative bias for the complete-case analyses was $14 \%$ for the estimate of current HRT use and 9\% for past HRT use. A single imputation of 'age at menopause' as 55 years gave only slightly attenuated estimates of risk for HRT use compared with the multiple imputation of 'age at menopause' (percentage relative bias of $-3 \%$ for current and $-4 \%$ for past users).

For 'age at menopause', the risks associated with an increase of 1 SD were similar for the complete-case analysis and the analysis with multiple imputation of 'age at menopause'. Both methods of single imputation of 'age at menopause' resulted in attenuated estimates of the risk for breast cancer (percentage relative bias was $-7 \%$ ).

\section{Discussion}

We used data from the MCCS to compare different methods for dealing with missing 'age at menopause' in analyses investigating the risk of HRT for invasive breast cancer in postmenopausal women. Our analyses showed that excluding women with 'age at menopause' missing from the analysis, (i.e. a complete-case analysis) resulted in higher estimates of the risk of HRT use for breast cancer compared with the estimates obtained from all postmenopausal women, irrespective of the inclusion or exclusion of 'age at menopause' in the model. The estimates of risk for HRT from single imputation of 55 years were slightly attenuated compared with the multiple imputation estimates. The differences between the single imputation of 55 years' estimates and the multiple imputation estimates were small compared with the higher estimates observed for the complete-case analysis. Although we agree with Pike et al. ${ }^{3}$ and Rockhill et al. ${ }^{4}$ that the methods of single imputation of 'age at menopause' give attenuated estimates of the risk of HRT, for our data the magnitude of this was small compared with that observed for estimates obtained from a complete-case analysis. The estimates of risk associated with an increase of $1 \mathrm{SD}$ in 'age at menopause' were attenuated for both methods of single imputation compared with multiple imputation.

Our imputation model assumed that women with 'age at menopause' missing (i.e. women with simple hysterectomy) were 'missing at random' and that the distribution of 'age at menopause' was similar to women with a natural menopause. Pike et al. ${ }^{3}$ proposed that a simple hysterectomy in some women may damage the blood supply to the ovaries, leading to an earlier cessation of ovarian function and thus an earlier 'age at menopause'. Literature to support this hypothesis is inconclusive. ${ }^{10-14}$ We compared the distribution of estradiol levels between women with an intact uterus and women with a simple hysterectomy from a randomly selected subcohort of the MCCS $^{15}$ and found no evidence to support the hypothesis of Pike et al. ${ }^{3}$

For the multiple imputations, we had assumed that the distribution of 'age at menopause' was Normally distributed. In fact, 'age at menopause' was slightly negatively skewed. This deviation from the assumption of Normality would be problematic if the aim of our analyses were to predict, say, the 5th or 95th percentiles of 'age at menopause' 16

We defined women with simple hysterectomy to be postmenopausal if their age at baseline was greater than 55 years and they did not have estradiol measured. Hence, the postmenopausal women selected for this paper do not represent all possible postmenopausal women in the MCCS and are over-represented in the 55 and above age group. This sampling bias is a problem for all breast cancer cohorts where the analysis is restricted to postmenopausal women. In the paper by Rockhill et al., 4 which also investigated bias in breast cancer analyses, the dataset only included women with simple hysterectomy who were older than the age at which natural menopause had occurred in $90 \%$ of the cohort (54 years for current cigarette smokers and 56 years for nonsmokers). In the MWS, ${ }^{2}$ women with a simple hysterectomy were defined as postmenopausal if they were aged 53 years or older.

'Age at menopause' was unknown for approximately $17 \%$ of the women in this study. Other cohort studies may have a smaller proportion of women with 'age at menopause' missing, possibly resulting in smaller differences in the estimates of risk between a completecase analysis and analyses including all women. Age at menopause was unknown for $11 \%$ of all women in the pooled analysis of 50 epidemiological studies. ${ }^{1}$ For the 


\begin{tabular}{|c|c|c|c|}
\hline Predictor variable & Hazard ratioa & $95 \% \mathrm{Cl}$ & Relative percentage change ${ }^{b}$ \\
\hline \multicolumn{4}{|c|}{$\begin{array}{l}\text { Model } 1 \text { ( } n=11035 ; 328 \text { cases) } \\
\text { (without adjustment for 'age at menopause') }\end{array}$} \\
\hline Never user & 1.00 & & \\
\hline Past user & 1.27 & $0.90-1.80$ & \\
\hline Current user & 1.90 & $1.44-2.51$ & \\
\hline \multicolumn{4}{|c|}{$\begin{array}{l}\text { Model } 2(n=11035 ; 328 \text { cases }) \\
\text { (complete-case analysis) } \\
\text { HRT }\end{array}$} \\
\hline Never user & 1.00 & & \\
\hline Past user & 1.28 & $0.90-1.81$ & $9 \%$ \\
\hline Current user & 1.93 & $1.46-2.55$ & $14 \%$ \\
\hline Age at menopause & 1.20 & $1.06-1.35$ & $-2 \%$ \\
\hline \multirow{2}{*}{\multicolumn{4}{|c|}{$\begin{array}{l}\text { Model } 3 \text { ( } n=13357 ; 396 \text { cases) } \\
\text { (without adjustment for 'age at menopause') } \\
\text { HRT }\end{array}$}} \\
\hline & & & \\
\hline Never user & 1.00 & & \\
\hline Past user & 1.13 & $0.83-1.56$ & \\
\hline Current user & 1.65 & $1.29-2.12$ & \\
\hline \multicolumn{4}{|c|}{$\begin{array}{l}\text { Model } 4 \text { ( } n=13357 \text {; } 396 \text { cases) } \\
\text { (multiple imputation of 'age at menopause') }\end{array}$} \\
\hline Never user & 1.00 & & \\
\hline Past user & 1.17 & $0.85-1.61$ & \\
\hline Current user & 1.69 & $1.31-2.17$ & \\
\hline Age at menopause & 1.21 & $1.08-1.36$ & \\
\hline \multicolumn{4}{|c|}{$\begin{array}{l}\text { Model } 5 \text { ( } n=13357 ; 396 \text { cases) } \\
\text { (single imputation of } 55 \text { years for 'age at menopause') } \\
\text { HRT }\end{array}$} \\
\hline Never user & 1.00 & & \\
\hline Past user & 1.12 & $0.82-1.55$ & $-4 \%$ \\
\hline Current user & 1.64 & $1.28-2.11$ & $-3 \%$ \\
\hline Age at menopause ${ }^{c}$ & 1.13 & $1.01-1.26$ & $-7 \%$ \\
\hline \multirow{2}{*}{\multicolumn{4}{|c|}{$\begin{array}{l}\text { Model } 6 \text { ( } n=13 \text { 073; } 393 \text { cases) } \\
\text { (single imputation by equating age at } \\
\text { hysterectomy to 'age at menopause') } \\
\text { HRT }\end{array}$}} \\
\hline & & & \\
\hline Never user & 1.00 & & \\
\hline Past user & 1.18 & $0.86-1.62$ & $1 \%$ \\
\hline Current user & 1.71 & $1.33-2.19$ & $1 \%$ \\
\hline Age at menopause & 1.13 & $1.03-1.24$ & $-7 \%$ \\
\hline
\end{tabular}

aAll models include adjustment for education level, age at menarche, age at first live birth and parity (combined variable), smoking status, alcohol intake, body mass index and ethnic group.

bRelative percentage change from the multiple imputation estimate.

cHazard ratio for 'age at menopause' represents the risk associated with an increase of 1 standard deviation (i.e. 4.8 years).

HRT, hormone replacement therapy.

Nurses Health Study ${ }^{17}$ and the MWS ${ }^{2}$ the percentage of women with 'age at menopause' unknown was not reported. 'Age at menopause' was only a weak confounder in our cohort but may be a stronger confounder in other studies, possibly leading to larger differences in the estimates of risk of HRT between the different methods of imputation. In the pooled analysis of 50 epidemiological studies, ${ }^{1}$ failure to adjust for 'time since menopause' would have underestimated the relative risk associated with each year of use in current users of HRT (unadjusted percentage increase in relative risk of $0.8 \%$ vs adjusted percentage increase in relative risk of $2.3 \%$ ).

Our findings showed that women with 'age at menopause' missing did not represent a random sample of the population, and that complete-case analyses resulted in higher estimates of the risk of HRT for breast cancer. We recommend that analyses investigating the association between HRT and breast cancer should present the results in two ways: excluding the women with 'age at menopause' missing and including the women with 'age at menopause' missing using multiple imputation. For both methods, the estimates of risk for breast cancer, with and without the adjustment of 'age at menopause', should be given.

\section{Acknowledgements}

The MCCS was made possible by the contribution of many people, including the original investigators and the diligent team who recruited the participants and who continue working on follow-up. The authors would also like to express their gratitude to the many thousands of Melbourne residents who continue to participate in the study.

Statements on funding and competing interests

Funding Cohort recruitment was funded by VicHealth and The Cancer Council Victoria. This study was funded by grants from the National Health and Medical Research Council (126402; 209057; 170215; 251533) and VicHealth (1999-0227; 1998-0406) and was further supported by infrastructure provided by The Cancer Council Victoria.

Competing interests None identified.

References

1 Breast cancer and hormone replacement therapy: collaborative reanalysis of data from 51 epidemiological studies of 52,705 women with breast cancer and 108,411 women without breast cancer. Collaborative Group on Hormonal Factors in Breast Cancer. Lancet 1997; 350: 1047-1059.

2 Million Women Study Collaborators. Breast cancer and hormone-replacement therapy in the Million Women Study. Lancet 2003; 362: 419-427.

3 Pike MC, Ross RK, Spicer DV. Problems involved in including women with simple hysterectomy in epidemiological studies 
measuring the effects of hormone replacement therapy on breast cancer risk. Am J Epidemiol 1998; 147: 718-721.

4 Rockhill B, Colditz GA, Rosner B. Bias in breast cancer analyses due to error in age at menopause. Am J Epidemiol 2000; 151: 404-408.

5 Lyles $\mathrm{RH}$, Allen AS. Estimating crude or common odds ratios in case-control studies with informatively missing exposure data. Am J Epidemiol 2002; 155: 274-281.

6 Demissie S, LaValley MP, Horton NJ, Glynn RJ, Cupples LA. Bias due to missing exposure data using complete-case analysis in the proportional hazard regression model. Stat Med 2003; 22: 545-557.

7 Barzi F, Woodward M. Imputations of missing values in practice: results from imputations of serum cholesterol in 28 cohort studies. Am J Epidemiol 2004; 160: 34-45.

8 Giles GG, English DR. The Melbourne Collaborative Cohort Study. IARC Sci Publ 2002; 156: 69-70.

9 Carlin JB, Li N, Greenwood P, Coffey C. Tools for analysing multiple imputed datasets. The Stata Journal 2003; 3: 226-244.

10 Bhattacharya S, Mollison J, Pinion S, Parkin DE, Abramovich $\mathrm{DR}$, Terry $\mathrm{P}$, et al. A comparison of bladder and ovarian function two years following hysterectomy or endometrial ablation. $\mathrm{Br} J$ Obstet Gynaecol 1996; 103: 898-903.

11 Chalmers C, Lindsay M, Usher D, Warner P, Evans D, Ferguson M. Hysterectomy and ovarian function: levels of follicle stimulating hormone and incidence of menopausal symptoms are not affected by hysterectomy in women under age 45 years. Climacteric 2002; 5: 366-373.

12 Derksen JG, Brolmann HA, Wiegerinck MA, Vader HL, Heintz
AP. The effect of hysterectomy and endometrial ablation on follicle stimulating hormone (FSH) levels up to 1 year after surgery. Maturitas 1998; 29: 133-138.

13 Bukovsky I, Halperin R, Schneider D, Golan A, Hertzianu I, Herman A. Ovarian function following abdominal hysterectomy with and without unilateral oophorectomy. Eur J Obstet Gynecol Reprod Biol 1995; 58: 29-32.

14 Siddle N, Sarrel P, Whitehead M. The effect of hysterectomy on the age at ovarian failure: identification of a subgroup of women with premature loss of ovarian function and literature review. Fertil Steril 1987; 47: 94-100.

15 Simpson JA, Maclnnis RJ, English DR, Gertig DM, Morris HA, Giles GG. A comparison of estradiol levels between women with a hysterectomy and ovarian conservation and women with an intact uterus. Climacteric 2005; 8: 300-303.

16 Schafer JL. Multiple imputation: a primer. Stat Methods Med Res 1999; 8: 3-15.

17 Colditz GA, Hankinson SE, Hunter DJ, Willett WC, Manson JE, Stampfer MJ, et al. The use of estrogens and progestins and the risk of breast cancer in postmenopausal women. $N$ Engl $J$ Med 1995; 332: 1589-1593.

18 Van Buuren S, Boshuizen HC, Knook DL. Multiple imputation of missing blood pressure covariates in survival analysis. Stat Med 1999; 18: 681-694

19 Trichopoulos D, MacMahon B, Cole P. Menopause and breast cancer risk. J Natl Cancer Inst 1972; 48: 605-613.

20 Rubin DB. Multiple Imputation for Nonresponse in Surveys. New York, NY: John Wiley, 1987.

21 Schafer JL. Analysis of Incomplete Multivariate Data. London, UK: Chapman \& Hall, 1997.

\section{APPENDIX}

The multiple imputation procedure consisted of the five steps outlined below.

Step 1: An imputation model was devised using the recommendations of Van Buuren et al. 18 The model included all of the predictor variables and the outcome variable, diagnosis of breast cancer (yes/no). Follow-up time was not included since the majority of women (92\%) were followed up to 1 June 2002 with no diagnosis of breast cancer. The data on 'age at menopause' were assumed to be 'missing at random'. 'Missing at random' means that the probability of the data being missing may depend on any of the observed variables but is not dependent on any unobserved variable. 16

For the imputation model, women with a bilateral oophorectomy were excluded. These women have an earlier 'age at menopause' compared with women who have had a natural menopause, resulting in a lower risk of breast cancer. ${ }^{19} \mathrm{We}$ assumed that the distribution of 'age at menopause' for women with a simple hysterectomy was the same as for women with a natural menopause.

Step 2: The imputation model was used to generate five sets of imputed values for the missing data points thus creating five completed datasets. Because Rubin 20 has shown that there is little benefit from using more than five to ten imputations and for our proportion of missing data $(<20 \%)$, we considered that five imputations were adequate. Data were imputed from the posterior predictive distribution of the missing data given the observed data. The posterior predictive distribution of the data was assumed to be multivariate Normal, so that all of the predictor variables (categorical and continuous) were assumed to be Normally distributed. This assumption is reasonable when no values are missing for the categorical predictor variables, as is the case for our data. 21 The Expectation-Maximisation (EM) algorithm (a likelihood-based approach to handling missing data) was applied first to obtain maximum likelihood estimates that were used as starting values for the data augmentation step. Data augmentation first performs a random imputation of the missing data by assuming the parameters of the posterior predictive distribution equal the maximum likelihood estimates from the EM algorithm. New parameters are then drawn from the posterior distribution of the observed and imputed data. This procedure is repeated many times creating a Markov Chain that eventually converges. Convergence of the means and standard deviations of 'age at menopause' to their posterior distribution was assessed by examination of time-series and autocorrelation function plots. ${ }^{21}$

Step 3: The imputed values generated in Step 2 using multiple imputation were imputed from the following conditional distributions:

(a) 'Age at menopause' must be less than or equal to 'age at first hormone replacement therapy (HRT) use plus 12 months' for women whose age at first HRT was known and not before age at hysterectomy $(n=771)$.

(b) 'Age at menopause' must be greater than or equal to the 'age at hysterectomy' for women who had a simple hysterectomy and the age at hysterectomy was known $(n=2038)$.

The distribution of 'age at menopause' for the imputed values was compared with the observed distribution (excluding women who had had a bilateral oophorectomy).

Step 4: For each of the five completed datasets (including women who had had a bilateral oophorectomy), a Cox proportional hazard regression was performed (with attained age as the time axis and inclusion of all predictor variables), from which the estimate of $\theta_{i}$ (log of the hazard ratio) of interest and its estimated variance $\operatorname{var}\left(\theta_{i}\right)$ was obtained, for $i=1,2,3,4,5$.

Step 5: The results from the Cox proportional hazard regression modelling of the five different datasets were combined using the rules given by Rubin 20 to produce a multiple imputation estimate of $\theta$ (log of the hazard ratio) and $\operatorname{var}(\theta)$. The multiple imputation estimate of $\theta$ is simply the average of the $\theta_{i} \mathrm{~s}$, whereas the multiple imputation estimate of $\operatorname{var}(\theta)$ allows for the between- and withinimputation components of variation.

$\hat{\theta}=\frac{1}{m} \sum_{i=1}^{m} \hat{\theta}_{i}$

$\operatorname{vâr}(\hat{\theta})=\frac{1}{m} \sum_{i=1}^{m} \operatorname{vâr}\left(\hat{\theta}_{i}\right)+\left(1+\frac{1}{m}\right) \cdot\left(\frac{1}{m-1}\right) \sum_{i=1}^{m}\left(\hat{\theta}_{i}-\hat{\theta}\right)^{2}$,

where $m$ is the number of sets of imputed values. 